\title{
Mitochondrial involvement in tracheary element programmed cell death
}

\author{
X-H Yu ${ }^{1}$, TD Perdue ${ }^{1}$, YM Heimer ${ }^{1,2}$ and AM Jones ${ }^{\star, 1}$ \\ ${ }^{1}$ Department of Biology, The University of North Carolina at Chapel Hill, Chapel \\ Hill, NC 27599, USA \\ 2 Current address: The Jacob Blaustein Institute for Desert Research, Ben Gurion \\ University of the Negev, Sede Boger, 84990, Israel. \\ * Corresponding author: Alan M Jones, Department of Biology, CB\# 3280, Coker \\ Hall, The University of North Carolina at Chapel Hill, Chapel Hill, NC 27599- \\ 3280, USA. Tel: 919962 6932; Fax: 919962 1625; \\ E-mail: alan_jones@unc.edu
}

Received 25.5.01; revised 23.7.01; accepted 28.7.01

Edited by B Osborne

\begin{abstract}
The mitochondria pathway is regarded as a central component of some types of programmed cell death (PCD) in animal cells where specific signals cause the release of cytochrome $c$ from mitochondria to trigger a proteolytic cascade involving caspases. However, plant cells lack canonical caspases, therefore a role for the mitochondria in programmed cell death in plant cells is not obvious. Using plant cells which terminally differentiate, we provide evidence supporting the involvement of mitochondria in PCD, however the release of cytochrome cis insufficient to trigger the PCD. Prior to execution of cellular autolysis initiated by the rupture of the large central vacuole to release sequestered hydrolases, mitochondria adopt a definable morphology, the inner membrane depolarizes prior to death, and cytochrome $c$ is released from mitochondria. However, PCD can be blocked despite translocation of cytochrome $c$. These results suggest a role for the mitochondriain this PCD butdonotsupportthecurrentanimalmodelfora causative role of cytochrome $c$ in triggering PCD.

Cell Death and Differentiation(2002) 9, 189 - 198. DOI: 10.1038/sj/ $\mathrm{cdd} / 4400940$
\end{abstract}

Keywords: programmed cell death; mitochondria; cytochrome $c$; tracheary element; plant; betulinic acid

Abbreviations: BetA, betulinic acid; CsA, cyclosporin A; PCD, $\mathrm{CpD}$, cyclophilin $\mathrm{D}$; programmed cell death; PT, permeability transition; TUNEL, terminal deoxynucleotidyl transferase-mediated dUTP nick end labeling; VDAC, voltage-dependent anion channel

\section{Introduction}

The most widely studied form of programmed cell death (PCD) in animal cells is apoptosis. Apoptosis is characterized by a distinct set of morphological and biochemical features, including cell shrinkage, cytoplasmic membrane blebbing, lobing of the nucleus, nuclear DNA fragmentation, and disassembly into apoptotic bodies. ${ }^{1}$ Participation of mitochondria in apoptosis is based on observations using a cell free system for apoptosis, ${ }^{2}$ on immunolocalization of apoptotic and anti-apoptotic factors to the outer mitochondrial membrane, ${ }^{3}$ and on measurements of mitochondrial membrane function in relation to death. ${ }^{4}$ Furthermore, studies indicated that proteins in the Bcl-2 family affect the release of cytochrome $c$ from mitochondria ${ }^{5}$ which subsequently triggers a proteolytic cascade leading to PCD. ${ }^{6}$ These specialized proteases, called caspases, are found in C. elegans, Drosophila and mammals, but no canonical forms are found in fungi, protists, and plants. A current hypothesis is that cytochrome $c$ binds to the scaffold complex called apoptotic protease activation factor 1 (Apaf1), and causes recruitment of procaspase-9 which transactivates due to close proximity. ${ }^{7}$ Clearly, cytochrome $c$, Apaf1, and procaspase 9 do not define the entire apparatus, since other apoptosis inducing factors (e.g. AIF, diablo) play key roles in the mitochondrial pathway. ${ }^{8,9}$

There are at least two mechanisms in animal cells for the release of cytochrome $c$ into cytoplasm and both require active participation of the mitochondria. ${ }^{10,11}$ The first mechanism occurs directly via the voltage-dependent anion channel (VDAC) but details are presently scant. ${ }^{5}$ The second mechanism occurs indirectly via the rupture of the outer membrane caused by water influx into the matrix through a transient permeability transition pore (PTP). ${ }^{12}$ PTP functions as a calcium-, voltage-, $\mathrm{pH}$-, and redox-gated channel with several levels of conductance but poor ion selectivity. Continual PTP opening permits the release of solutes from matrix with molecular masses up to $1500 \mathrm{Da}^{13}$ The PTP is thought to be composed of several proteins including hexokinase, the VDAC present on the outer membrane, the adenine nucleotide translocator (ANT) present on the inner membrane, and the matrix-localized protein, cyclophilin $D$ $(\mathrm{CpD})$. Some anticancer drugs and other chemicals, such as betulinic acid (BetA) and cyclosporin A (CsA), can trigger or block PTP opening and cytochrome $c$ release, thus inducing or inhibiting apoptosis, respectively.

The second mechanism relies on matrix swelling causing mechanical disruption of the outer mitochondrial membrane and therefore this mechanism is easily identified by the mitochondrial ultrastructure. Opening of PTP leads to equilibration of solutes across mitochondrial membranes and results in mitochondrial membrane depolarization followed by large amplitude matrix swelling. Rupture is caused by the increase in the colloidosmotic pressure. $\mathrm{Ca}^{2+}$ can increase permeability of the inner membrane to small solutes, thus causing osmotic swelling of the mitochondria matrix and physical disruption of the outer membrane. ${ }^{14}$ The open and closed state of the PTP, and thus the 
potential release of cytochrome $c$, are coupled to cellular metabolism and survival factors. ${ }^{15}$

Other models that utilize the former mechanism propose formation of pores for cytochrome $c$ release which can happen in a very subtle way leaving much of the mitochondrial structure and function intact. It has been proposed that members of the Bcl-2 family of apoptosis controlling proteins can form channels in the outer membrane or alternatively cause membrane instability that leads to cytochrome $c$ release. ${ }^{16} \mathrm{Bax}$, a pro-apoptotic member of this family, may regulate the size of the VDAC to allow cytochrome $c$ translocation. ${ }^{5,17}$

PCD is essential throughout the life cycle of plants. It is involved in the development of aleurone and root cap cells, tracheary element (TE) formation, somatic embryogenesis, senescence and plant-pathogen interaction. ${ }^{18}$ However little is known about its mechanism. Much of the concept of PCD in plants derives from the sole observation of DNA fragmentation in cells, a feature of both animal and plant PCD. ${ }^{19}$ In plants, nuclear DNA degradation can be detected by DNA ladders and in single cells with terminal deoxynucleotidy transferase-mediated dUTP nick end labeling (TUNEL). ${ }^{20,21}$

Perhaps our best understanding of the mechanism of a plant $P C D$ is one which occurs during the terminal differentiation of a tracheary element (TE). A TE is a functional cell corpse that forms a single unit of the waterconducting vessels in xylem tissue. TE formation is a classic example of terminal differentiation involving the coordination between the formation of a secondary cell wall and autolysis of its protoplasm. ${ }^{21}$ Groover et al. ${ }^{20,21}$ characterized the morphological changes associated with TE formation in suspension-cultured mesophyll cells isolated from zinnia leaves. Cells expand $24 \mathrm{~h}$ after isolation, then synthesize a secondary cell wall that provides the rigid structure of the vessels. The cell prepares for autolysis by nascent synthesis of hydrolases which become sequestered in its large central vacuole well before the wall becomes apparent. A regulatory protease is secreted during wall synthesis, and when its level of activity reaches a threshold level, $\mathrm{Ca}^{2+}$ influx is triggered and the vacuole ruptures to release its hydrolases. Inhibitors of calcium release block this penultimate event of TE PCD. Hydrolases clear the protoplasm, leaving behind a hollow corpse.

There are few studies on the possible involvement of cytochrome $c$ or the mitochondrion in a plant PCD. Stein and Hansen $^{22}$ have shown that D-mannose-induced cell death is associated with cytochrome $c$ release in maize cells. Cytochrome $c$ was found to be translocated from the mitochondria to the cytosol during heat-induced PCD in cucumber plants, ${ }^{23}$ and in menadione-induced death in tobacco protoplasts. ${ }^{24}$ However, no cytochrome $c$ released from mitochondria was observed in the PCD during pollination-induced petal senescence in petunia. ${ }^{25}$ Wen and Chase $^{26}$ have shown that changes in mitochondrial gene expression occur prior to pollen cell death. Thus, while examples are relatively few, evidence is accumulating to support a role for the mitochondria in PCD.

In order to investigate the role of mitochondria in the PCD occurring in the terminal differentiation of TEs, we examined the morphology of mitochondria and the translocation of cytochrome $c$. We have manipulated PCD and cytochrome $c$ with various agents in order to determine whether PCD and cytochrome $c$ release are strictly coordinated. While changes in mitochondria morphology and function were observed and suggest a role for mitochondria in TE PCD, the current evidence suggests that cytochrome $c$ release is not sufficient for induction of the PCD in TEs. Thus, we conclude that while the mitochondria do play a regulatory role in TE PCD, the mechanism is distinct from the current model for the mitochondrion as a central executioner in animal cells. ${ }^{7}$

\section{Results \\ Calcium induction of cytochrome $c$ release from mitochondria}

We have previously shown that calcium mobilization is necessary and sufficient to trigger TUNEL in TEs that are competent to execute a PCD. ${ }^{21}$ Calcium mobilization is also required for $P C D$ in most animal cells. $\mathrm{Ca}^{2+}$ triggers mitochondria to undergo a permeability transition to release apoptogenic proteins such as cytochrome $c$, procaspase 9 or AIF from the intermembrane space into cytosol. ${ }^{12}$ Therefore, we tested whether $\mathrm{Ca}^{2+}$ mobilization triggers cytochrome $c$ release during TE maturaturation. When cultured cells were treated with $0.1 \mathrm{mM}$ of the calcium ionophore, A23187, the release of cytochrome $c$ in the cytosol was higher than that in untreated cells (Figure 1).

\section{Cytochrome $c$ released from mitochondria during TE formation}

As the per cent of TE undergoing PCD increased during maturation, the amount of cytochrome $c$ in the soluble fraction increased. This was determined by preparing fractions from

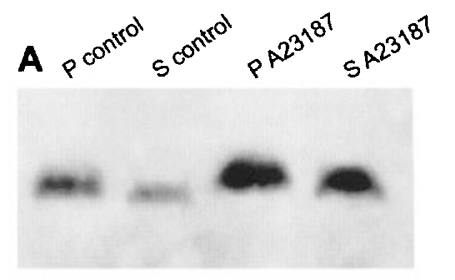

$\mathbf{B}$
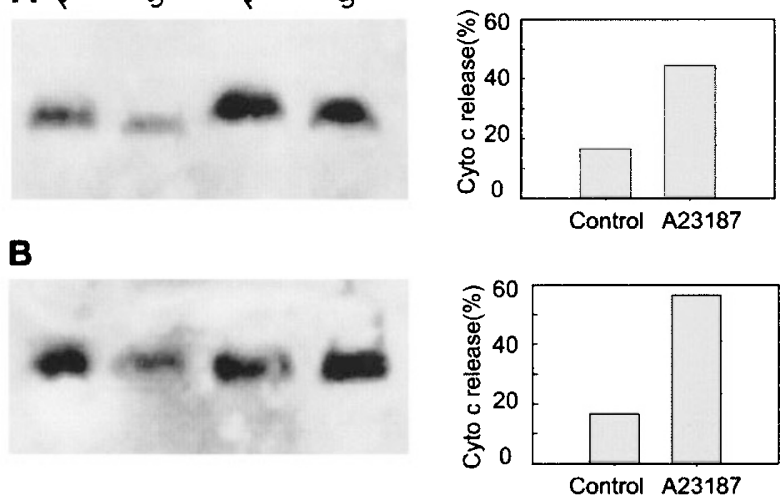

Figure 1 Calcium-induced release of cytochrome c. Cells ( $51 \mathrm{~h}$-old, i.e., approximately $20 \mathrm{~h}$ prior to when cells become TUNEL positive) were incubated with $0.1 \mathrm{mM} \mathrm{A23187}$, and were collected and washed after $2.5 \mathrm{~h}$ (A) and $5 \mathrm{~h}$ (B). Protein from cytosol and mitochondria was extracted separately, and analyzed by immunoblotting with a monoclonal cytochrome $c$ antibody. The signal was quantified and plotted as the $\%$ of total signal (pellet+soluble). P, pellet; S, supernatant 
48, 72 and $96 \mathrm{~h}$ cell cultures representing times prior to, at, and past the peak of death, respectively. Relative cytochrome $c$ content was determined by immunoblotting (Figure 2A). The ratio of cytochrome $c$ in the cytosol versus the total amount of cytochrome $c$ in mitochondria plus cytosol increased in parallel with the increase in per cent cells that were TUNEL positive (Figure 2B).

\section{Mitochondria morphology during TE maturation}

Differences between the morphology of mitochondria were observed between necrotic, non-necrotic, and terminallydifferentiating cells. The nature of the cell culture system enabled us to simultaneously study these three cell types. About $20 \%$ of the cells die during isolation (necrotic). The remaining mesophyll cells (non-necrotic) expand after $24 \mathrm{~h}$ from culture initiation, and begin to lay down secondary cell wall thickenings at about $72 \mathrm{~h}$ and execute a PCD (terminallydifferentiating). Death is defined here as the point in time when cytoplasmic streaming stops, which occurs instantaneously with the rupture of the large hydrolytic vacuole. ${ }^{20,21}$ After the vacuole ruptures, autolysis is rapid and complete within 2 h. At 24 h, non-necrotic mesophyll cells had an intact plasma membrane and tonoplast (vacuolar membrane), with chloroplasts appearing along the plasma membrane (Figure $3 A)$. The presence of tightly stacked grana of chloroplast was used as a marker for non-necrotic cells. Figure 3B shows that mitochondria in non-necrotic cells exhibited well organized ultrastructure. Specifically, the outer membrane was intact, the cristae were regularly organized, and the electron density
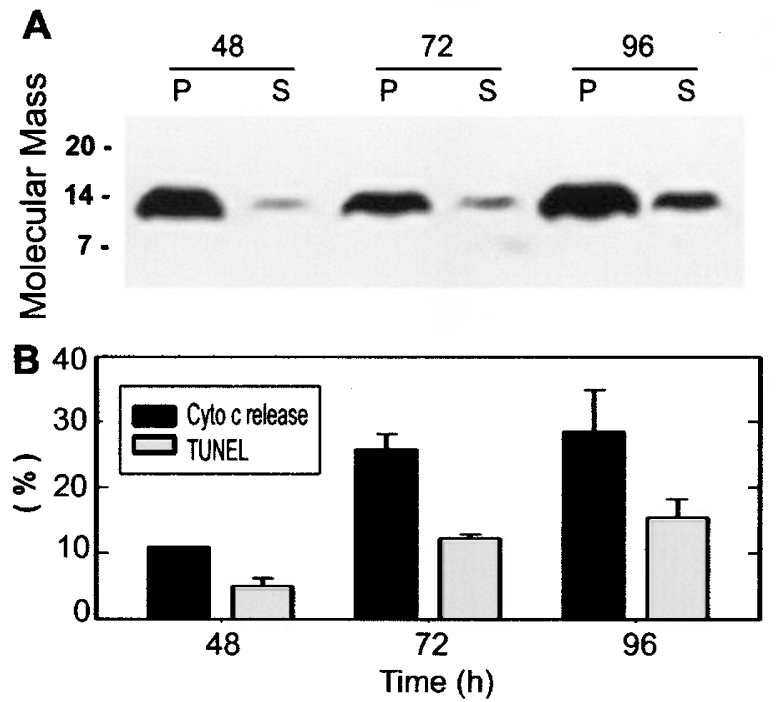

Figure 2 Translocation of cytochrome $c$ during TEs formation. Cells were collected at the indicated times (hours) after induction, protein from cytosol and mitochondria was separated and analyzed by immunoblotting with a monoclonal cytochdrome $c$ antibody as described in Materials and Methods (A). Molecular mass is indicated in kilodaltons. (B) The signal was digitized and plotted as a per cent of total cytochrome $c$ along with the per cent cells that were TUNEL positive at times indicated in A. P, pellet; S, supernatant. Error bar represents the standard error of the mean from two independent experiments of the matrix was even and similar to the cytoplasm. In contrast, necrotic cells had a plasma membrane detached from the cell wall (Figure $3 \mathrm{C}$ ). Mitochondria in these cells exhibited a more electron-dense matrix. The cristae appeared swollen in most cases with the internal membranes exhibiting a more angular contour when compared to non-necrotic cells (Figure 3D). Mitochondria in healthy non-differentiated, $72 \mathrm{~h}$ old cells exhibited similar ultrastructure as found in $24 \mathrm{~h}$-old non-necrotic cells (Figure 3E,F). Figure 4 illustrates the phenotypic changes occurring with mitochondria prior to death, as judged by the presence of an intact tonoplast. Most mitochondria in these cells contained an electron dense matrix but otherwise the ultrastructure remains intact (Figure $4 A, B$ ), although somewhat disorganized (discussed further below). In some differentiating cells that contained an intact
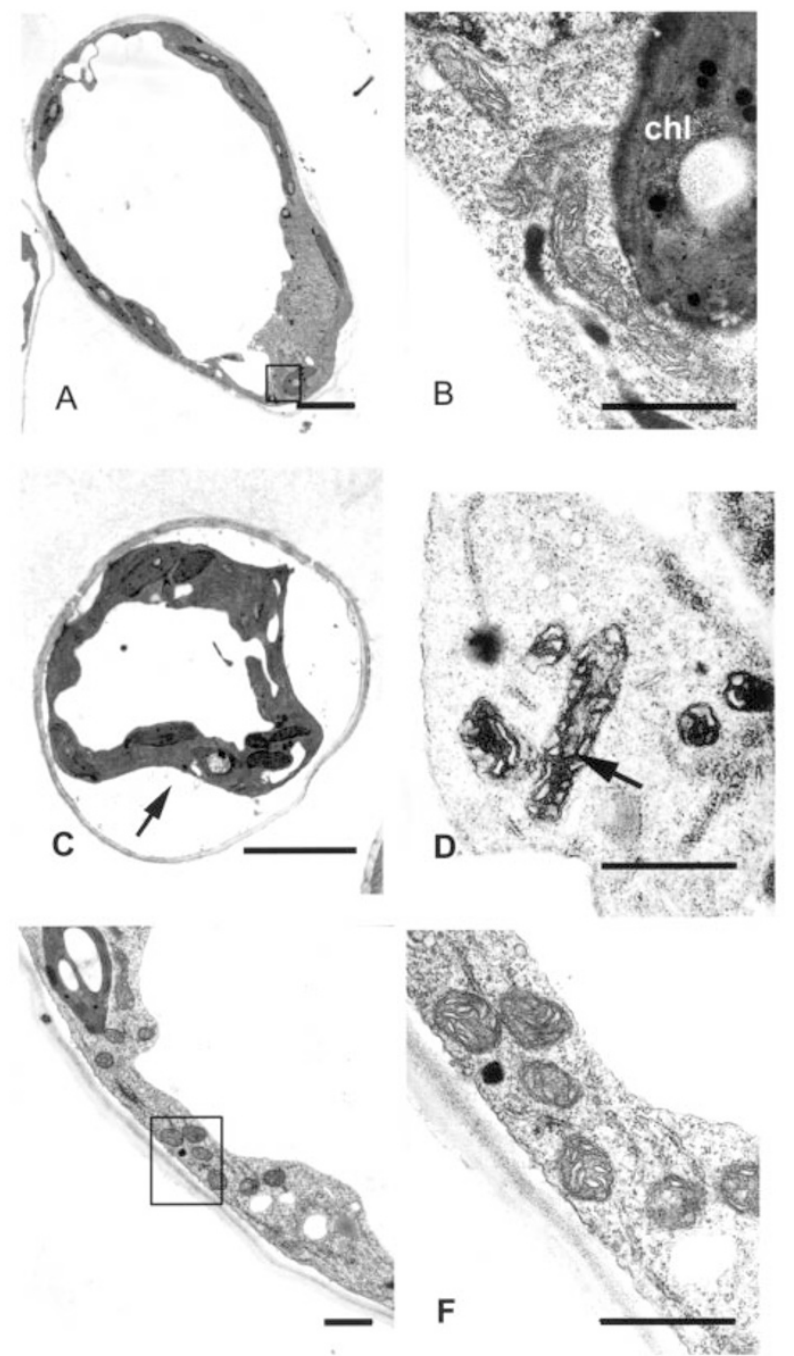

Figure 3 Ultrastructure of mitochondria in non-differentiating cells. In $24 \mathrm{~h}$ living cells (A, B), mitochondria have a well organized ultrastructure; A dead, $24 \mathrm{~h}$ cell has a detached plasma membrane (arrow) and the mitochondrial matrix is electron dense (C, D); At $72 \mathrm{~h}$, non-differentiating cells contain mitochondria that retain well organized ultrastructure $(\mathbf{E}, \mathbf{F})$. chl, chloroplast. Scale bar $=1 \mu \mathrm{m}$ in $\mathbf{B}, \mathbf{D}, \mathbf{F}$. Bar $=5 \mu \mathrm{m}$ in $\mathbf{A}, \mathbf{C}, \mathbf{E}$. Panels on the right represent higher magnifications of fields boxed within the image on the left 

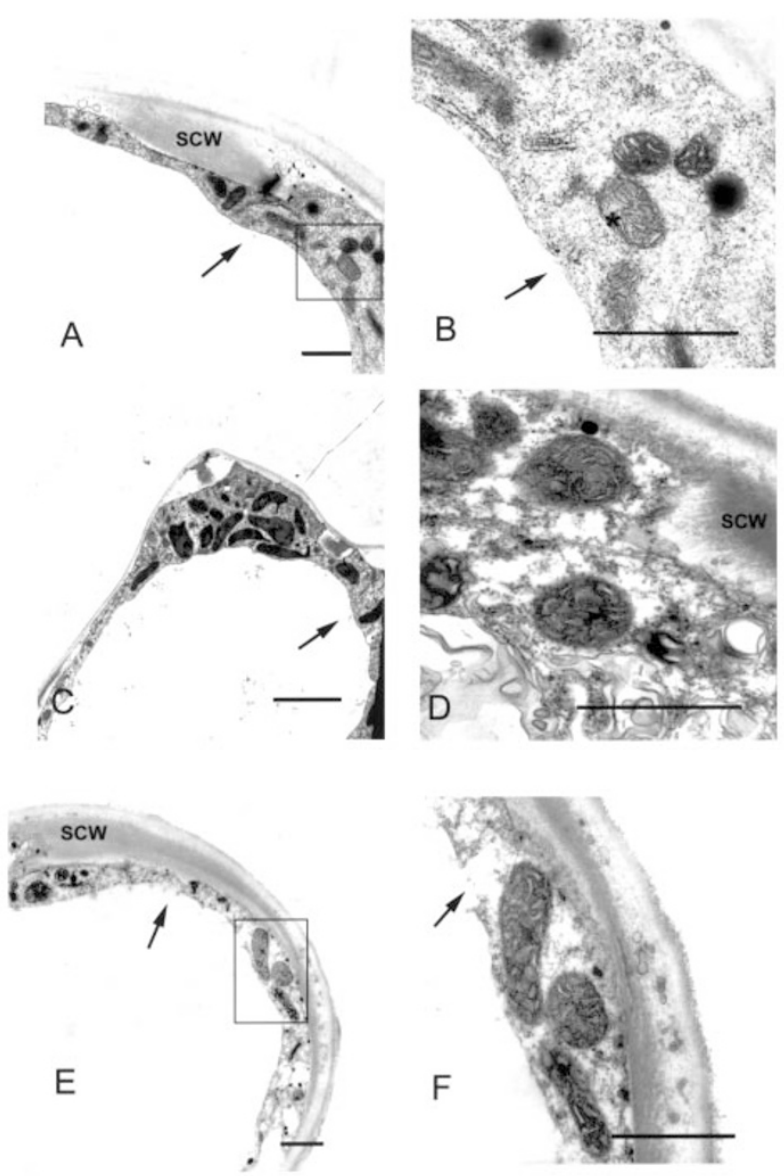

Figure 4 Ultrastructure of mitochondrial changes during TE formation Differentiating cells $(72 \mathrm{~h})$ are distinguished by their secondary cell wall. Their Golgi, ER and tonoplast remain intact (arrow) indicating that death has not been triggered (A). Some mitochondria exhibit unaltered, well organized ultrastructure ( ${ }^{*}$ in $\mathbf{B}$ ), while most exhibit electron-dense mitochondrial matrix (A, B). At a later stage of differentiation, the tonoplast (arrow) remains intact (C), but the cytoplasm appears less dense and the mitochondrial matrix is electron-dense (D). The tonoplast has lost its integrity in the cell shown in $\mathbf{E}$ and $\mathbf{F}$ (arrow). SCW, secondary cell wall. Scale bar $=1 \mu \mathrm{m}$ in $\mathbf{B}, \mathbf{D}, \mathbf{F}$; and $5 \mu \mathrm{m}$ in A, C, E. Panels on the right are higher magnifications of fields boxed within the corresponding panel on the left

tonoplast, the cytoplasm appeared granular, the mitochondrial matrix was more dense, and the cristae of the mitochondria were electron dense (Figure 4C,D). We interpret these cells to be at an advanced stage of TE maturation. When the tonoplast was broken, but well before autolysis was apparent, mitochondria lost their organized ultrastructure (Figure 4E,F).

The changes observed in mitochondria prior to the vacuole collapse are subtle but distinct from necrotic and non-necrotic cells. These differences are more apparent at higher magnification (Figure 5). The mitochondria shown in Figure 5B-D are from cells such as shown in Figure 3C. Compared to mitochondria from non-necrotic cells (bracket, Figure 5A), mitochondria in cells prior to the vacuole collapse have disorganized internal structure. The inner membrane system ranges from that containing swollen cristae (arrow a), and possibly an occasional discontinuity

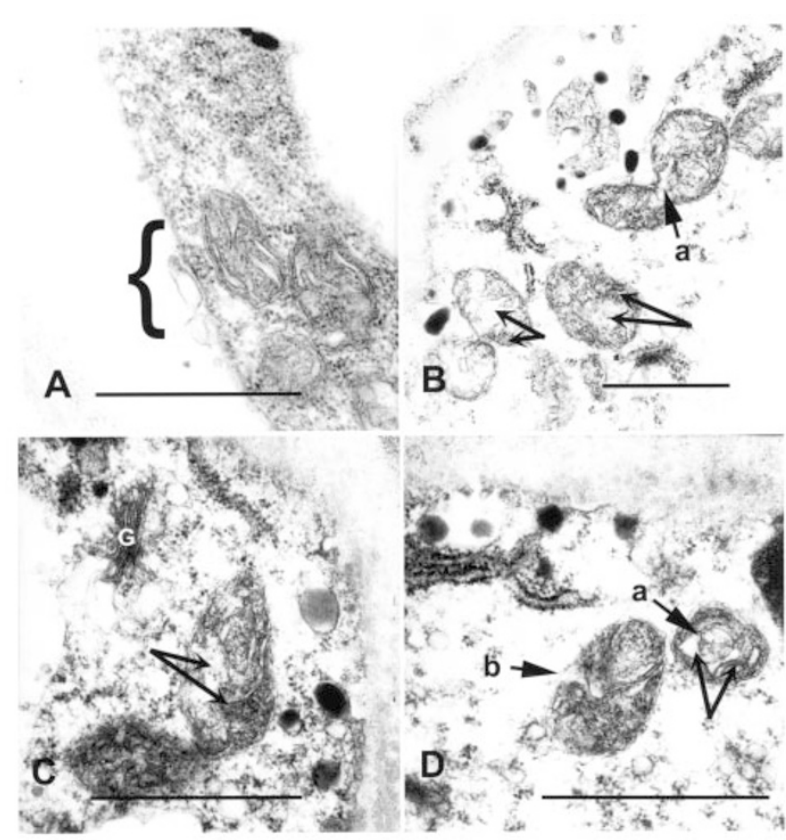

Figure 5 Ultrastructure of mitochondrial changes during TE differentiation. (A) Mitochondria (bracket) from a nondifferentiating cell have evenly electron dense matrix, cristae that are evenly spaced. (B-D). In contrast, mitochondria from cells prior to the collapse of the vacuole, have altered ultrastructure. Arrow a indicates swollen cristae, arrow $b$ indicates where the outer membrane may be discontinuous. Double arrows point to contrasting areas of density. G, Golgi Apparatus. Bars $=1 \mu \mathrm{m}$

in the outer membrane (arrow b). The matrix is unevenly electron dense (double arrows). Note the presence of the finely-preserved Golgi apparatus (G), indicating that the mitochondrial changes are not due to pervasive cytoplasmic degradation, nor to fixation artifact.

\section{Mitochondrial membrane depolarization during TE maturation}

Unlike in rat liver cells undergoing PCD where mitochondria morphology changes drastically before death, the mitochondria of TE change only subtly, although reproducibly. To investigate this further, we have utilized a voltage sensitive fluorescent dye, lipophilic cationic probe 5,5',6,6'-tetrachloro$1,1^{\prime}, 3,3^{\prime}$-tetraethylbenzimidazo-carbocyanine iodide (JC-1), to measure changes in the mitochondrial membrane potential $(\Delta \psi)$ that may precede death. Cells taken before PCD $(24 \mathrm{~h})$ and during PCD (72 h) were incubated with $10 \mu \mathrm{g} / \mathrm{ml} \mathrm{JC}-1$ for $20 \mathrm{~min}$, washed with fresh medium, and imaged by confocal microscopy. Most of the mitochondria in $24 \mathrm{~h}$-old, nonnecrotic cells appeared red (Figure 6A), indicating that mitochondrial membranes retained high voltage. In $72 \mathrm{~h}$-old cells, some of the mitochondria had lost their $\Delta \psi$, showing a mixture of red and green mitochondria (Figure 6B). In some cells, most of the mitochondria were green (Figure 6C, arrow a), indicating that they had depolarized (Figure 6D). After vacuole collapse, clearly indicated by cessation of cytoplasmic streaming, no mitochondria fluorescence was observed (Figure 6C, arrow b). Moreover, we conclude that the 

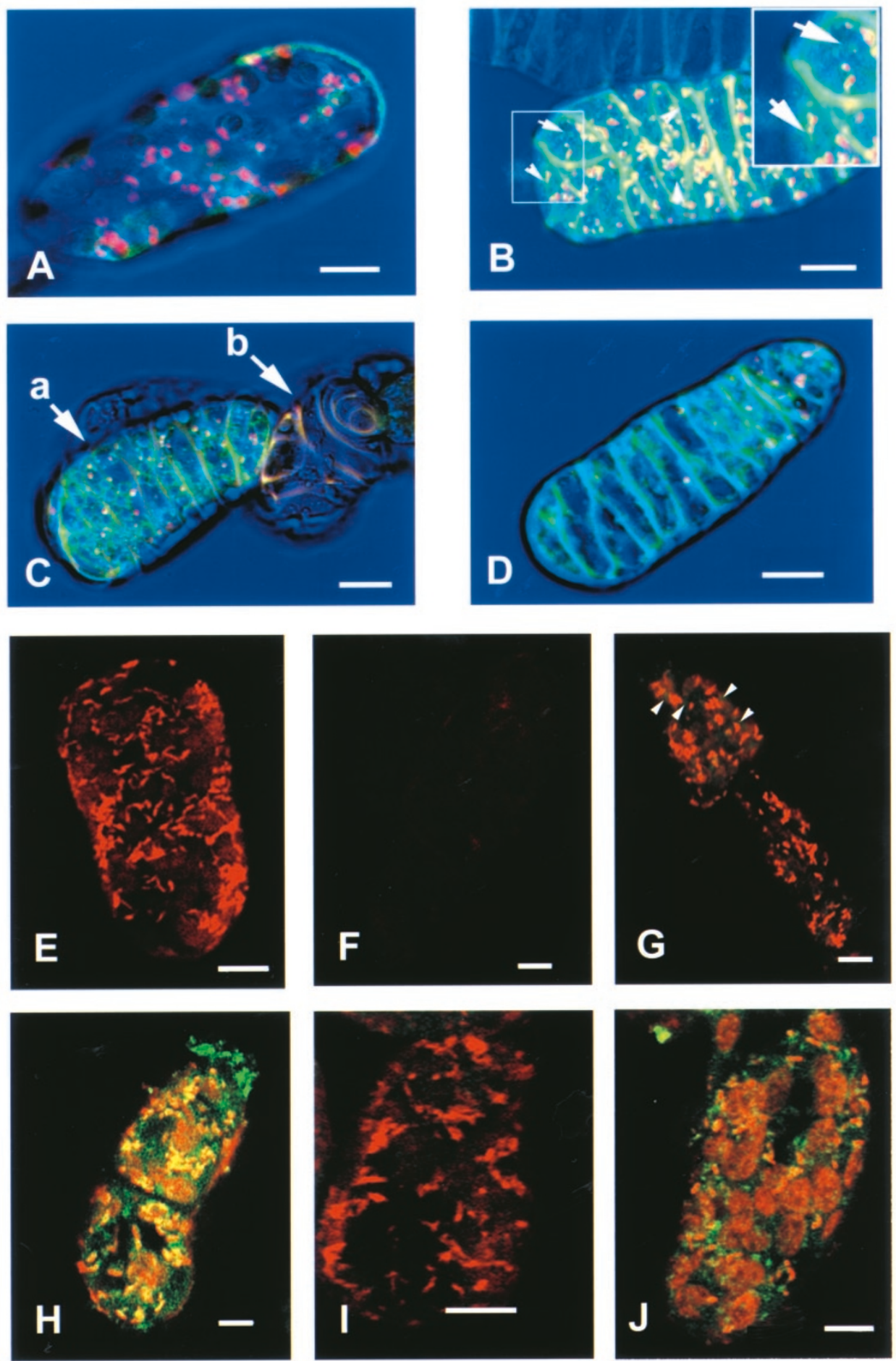

Figure 6 Mitochondrial depolarization during TE maturation. (A) Living cells incubated with JC-1 display red mitochondria indicating a hyperpolarized inner membrane. (B-D). Differentiating cells have a progressive increase in depolarized mitochondria. Arrowheads in B indicate the few depolarized mitochondria in an early stage whereas arrow a in $\mathbf{C}$ indicates a cell in which most of the mitochondria are depolarized. In $\mathbf{B}$, the boxed region on the left is magnified in the upper left inset. Arrow $b$ in $\mathbf{C}$ indicates a cell that is dead and completely autolyzed. In $(\mathbf{E}-\mathbf{J})$, cells (approximately $51 \mathrm{~h}$-old) were treated with various agents: $(\mathbf{E})$ control, untreated cells; (F) cells frozen prior to JC-1 staining; (G) $20 \mathrm{~min}$ of BetA $(20 \mu \mathrm{g} / \mathrm{ml})$ treatment, depolarized mitochondria are indicated by arrows; (H) $2 \mathrm{~h}$ BetA treatment, most mitochondria are depolarized. (I) BetA plus CsA, $2 \mathrm{~h}$, in most cells, CsA blocks the BetA-induced depolarization; (J) same as I, occasionally a cell is found in this treatment containing depolarized mitochondria. Scale bar $=10 \mu \mathrm{m}$ in $\mathbf{A}-\mathbf{D}$; and $5 \mu \mathrm{m}$ in $\mathbf{E}-\mathbf{J}$

observed mitochondrial depolarization preceded vacuole collapse (moment of death) because $\mathrm{JC}-1$ is unable to stain mitochondria after cell death (Figure 6F). This was shown by killing cells by rapid freezing (Figure 6) or aldehyde fixations 
(data not shown) and immediately assaying JC-1 staining of mitochondria.

\section{Mitochondrial membrane potential and cytochrome $c$ release}

The loss in mitochondrial membrane potential preceding the vacuole collapse suggests an active role in triggering TE death. One possible mechanism is the release of cytochrome $c$ via a voltage-dependent manner. To investigate this, we utilized betulinic acid (BetA) and cyclosporin A (CsA) to manipulate the membrane potential and monitored changes in PCD and cytochrome $c$ distribution. Figure $6 \mathrm{E}-\mathrm{J}$ shows the BetA and CsA effects on mitochondrial membrane potential. Figure 6G illustrates the rapid loss of $\Delta \psi$ in mitochondria treated with BetA. The change is more dramatic in mitochondria in cells after $2 \mathrm{~h}$ of BetA treatment (Figure $6 \mathrm{H}$ ). CsA blocks the BetA-induced depolarization in most cells (Figure 6I,J).

As shown in Figure 7A, BetA increased PCD as measured by TUNEL in a dose-dependent manner. As in some animal cells, ${ }^{27} \mathrm{CsA}$ blocked the BetA-induction of $\mathrm{PCD}$ in maturing TE (Figure 7B). Because high concentration of CsA alone has been reported to trigger $P C D$ in animal cells, ${ }^{27}$ we tested the effect of cells with $10 \mu \mathrm{M}$ CsA, and found no increase in PCD.

While BetA induces the collapse of $\Delta \psi$ and the release of apoptosis inducing factors in isolated mitochondria, ${ }^{6}$ it is not clear if the antagonistic effect of CsA on BetAinduced death occurs by blocking the BetA-induced cytochrome $c$ release. We tested this using maturing TEs. Figure $7 \mathrm{C}$ shows that while CsA dramatically inhibited BetA-induced PCD as measured by TUNEL, CsA had less inhibitory effect on the BetA-induced cytochrome $c$ translocation.

\section{Betulinic acid manipulation on mitochondria morphology}

As shown in Figure $8 \mathrm{~A}, \mathrm{~B}$, the mitochondria in $48 \mathrm{~h}$-old cells after $2 \mathrm{~h}$ BetA treatment exhibited a reduction in the internal membrane network and an increase in the mitochondrial matrix density, however, no dramatic mitochondria swelling was observed. This resembles the subtly-altered ultrastructure of mitochondria that occurs during TE maturation, suggesting that the mitochondrial morphology may be the consequence of membrane depolarization.

\section{Protease inhibitors block both the differentiation and cell death programs}

Arabidopsis lacks canonical caspases, although they do contain a small family of distantly-related genes called metacaspases, ${ }^{28}$ Thus, in the absence of classical effector caspases that are capable of activation by relocated cytochrome $c$, it is difficult to imagine how the animal model for the apoptosome-controlled death could operate in a plant cell. Since some animal PCD utilize noncaspase proteases to initiate death, ${ }^{29}$ we do not exclude the possibility that other proteases, including divergent proteases having caspase

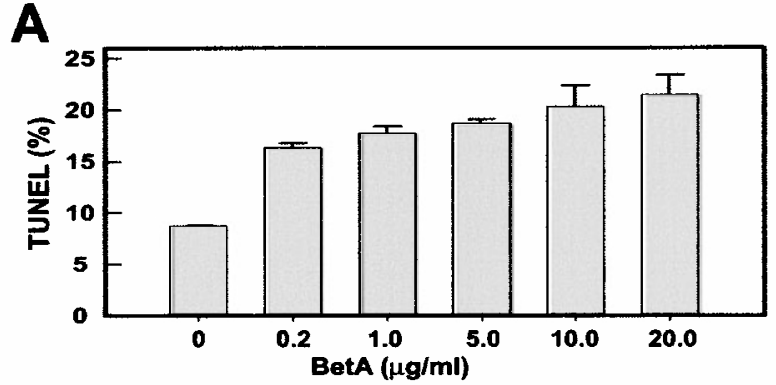

B
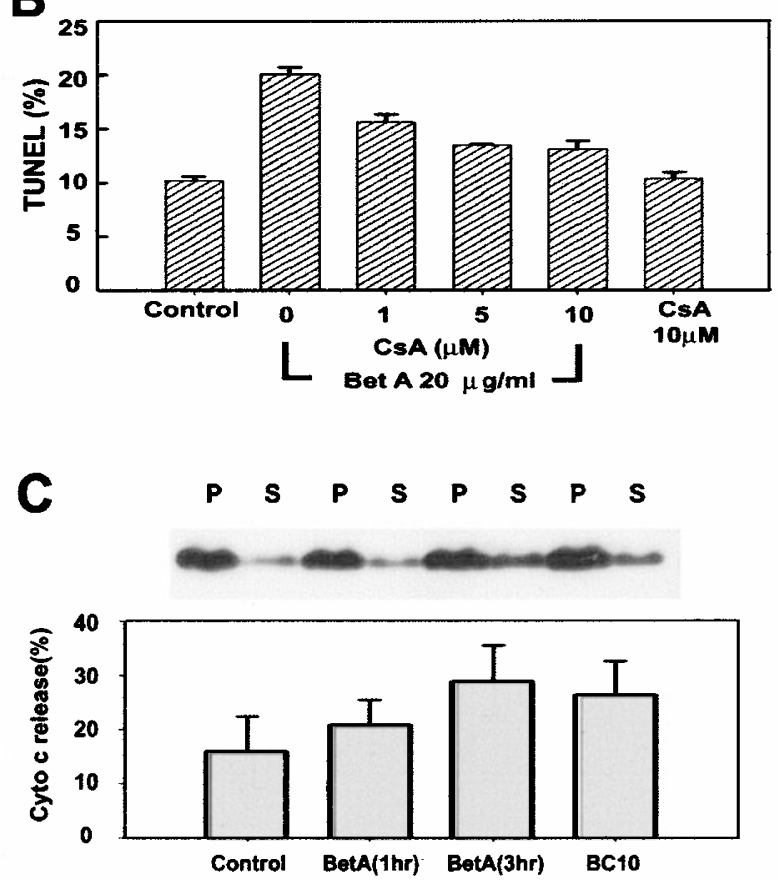

Figure 7 Betulinic acid and cyclosporin A effect on TE PCD and cytochrome $c$ translocation. (A) BetA $(20 \mu \mathrm{g} / \mathrm{ml}, 5 \mathrm{~h})$ induces cells to undergo DNA degradation as measured by TUNEL. The bar represents the standard error of the mean (S.E.M.) of three independent experiments. (B) CsA block cell TUNEL triggered by BetA. CsA at the indicated concentration was added to $51 \mathrm{~h}$-old cells just prior to $20 \mu \mathrm{g} / \mathrm{ml}$ BetA treatment, and coincubated for $5 \mathrm{~h}$, then TUNEL was assayed. Error bars represent the S.E.M. from duplicate experiments. (C) BetA and CsA effect on cytochrome $c$ release. Cells (51 hold) were incubated with $20 \mu \mathrm{g} / \mathrm{ml}$ BetA for 1 and $3 \mathrm{~h}$, and with both $20 \mu \mathrm{g} / \mathrm{ml}$ BetA and CsA $(10 \mu \mathrm{M})$ for $3 \mathrm{~h}(\mathrm{BC} 10)$. Control cells were mock treated for $3 \mathrm{~h}$. Cytochrome $c$ distributed between in the pellet and cytosol was indirectly measured as described in the Materials and Methods. The amount is presented as the \% of total (pellet+supernatant). Error bars represent the S.E.M. of three experiments

activity, may act as initiators or effectors of TE PCD. Three proteases activities are already proposed to be involved in TE $P C D^{21,30,31}$ and inhibitors to these were tested here for the ability to block TUNEL in developing TEs. At $48 \mathrm{~h}$ after induction (prior to cell wall synthesis), soybean trypsin inhibitor (STI), zFA-fmk and zVAD-fmk were added to cultures and the amount of TUNEL and \% TE formation was measured $48 \mathrm{~h}$ later (Figure 9 ). Trypsin, as shown before, ${ }^{21}$ prematurely induced TUNEL to levels observed during differentiation. Soybean trypsin inhibitor dramatically reduced TUNEL, 

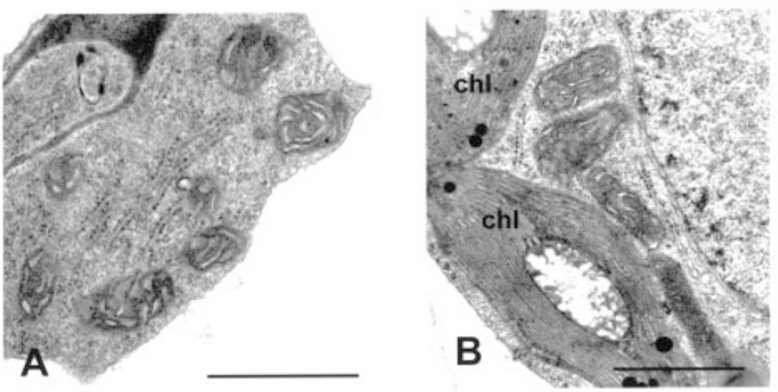

Figure 8 Betulinic acid (BetA) effect on mitochondria ultrastructure. BetA $(20 \mu \mathrm{g} / \mathrm{ml}$, final) was added to $48 \mathrm{~h}$-old cells and incubated for $2 \mathrm{~h}$, the mitochondria exhibited a reduction in the internal membrane network and an increase in the mitochondrial matrix density $(\mathbf{A}, \mathbf{B})$. chl: chloroplast. Standard bar $=1 \mu \mathrm{m}$

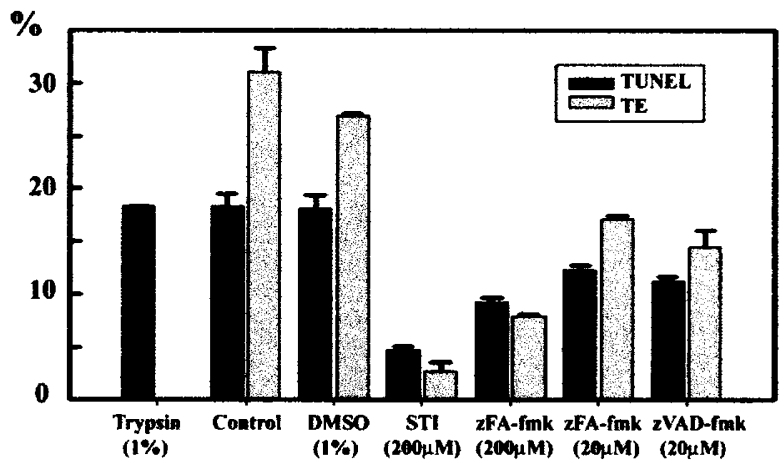

Figure 9 Protease inhibitor effect on TUNEL and TE formation. z-VAD-fmk (in DMSO), z-FA-fmk (in DMSO) and STI at the indicated concentration was added to $48 \mathrm{~h}$-old cells and culture continued for another 2 days, TUNEL and TEs percentage were assayed. Cells with no treatment and 1\% DMSO were used as control. 1\% trypsin was used as a positive control, and cells for TUNEL assay were collected after $6 \mathrm{~h}$ treatment. Error bars represent duplicate samples in one experiment. This experiment was repeated with the same results

whereas the cathepsin B inhibitor, zFA-fmk, and pancaspase inhibitor, zVAD-fmk had less effect.

\section{Discussion}

\section{Mitochondria are involved in TE PCD}

Mitochondria play a role in apoptotic and nonapoptotic animal cell death in at least three ways: (1) disruption of electron transport and oxidative phosphorylation, (2) release of apoptotic inducing factors or other catabolic degradative processes, and (3) alteration of cellular reduction-oxidation potential. $^{12}$ However, conservation of the mitochondrial pathway or its components between evolutionarily diverse organismal groups such as metazoans and plants has not been examined, and therefore it is not known which, if any, of these effects are shared. Conservation of mechanism provides insight on the primordial mitochondrial pathway.

Our study focuses on the possible role of mitochondria in a plant PCD. We chose to examine the PCD of terminally-differentiating TEs because of its importance in plant development and because it is a well-characterized system for a plant PCD. We found changes in the electron density of the mitochondrial matrix and mitochondrial ultrastructure prior to the trigger of death marked by the collapse of the vacuole. The use of a membrane potential dye also enabled us to conclude that the mitochondrial changes occur prior to the trigger of death. The ordering of events is possible because the membrane potential change occurred prior to vacuole collapse. We observed a clear depolarization of the mitochondrial membrane, thus conclude that this event precedes death. These results indicate that mitochondria are involved in the PCD of TEs, which is consistent with the hypothesis that mitochondrial dysfunction is a conserved feature of PCD in animal and plants. ${ }^{11}$

The changes in mitochondrial morphology of TE are clearly different from changes in many animal cells where the matrix swells and the outer membrane ruptures. These are caused by opening of the PTP or by altered polarization of the inner mitochondrial membrane. However, mitochondrial swelling is not observed in all instances of apoptosis, ${ }^{32,33}$ and cytochrome $c$ release from mitochondria can occur without major changes in mitochondrial ultrastructure. ${ }^{34}$ In these instances, release of apoptotic factors is thought to occur without sustained formation of a PTP, because CsA is unable to block death.

\section{Mitochondria permeability transition and cytochrome $c$ release in TE PCD}

BetA acid is a novel anticancer drug with specificity for neuroectodermal tumors. ${ }^{35}$ It induces PCD via a direct effect on mitochondria in a number of systems, including TE differentiation as shown here. In a cell free system, BetA provokes a dissipation of the mitochondrial transmembrane potential, induces mitochondrial permeability transition, and releases soluble factors such as cytochrome $c$ or apoptosis-inducing factor into cytoplasm to mediate the cleavage of caspase-8, caspase- 3 and nuclear fragmentation. ${ }^{6}$ In our cell culture system, BetA triggered mitochondrial depolarization, cytochrome $c$ release and triggered TUNEL, suggesting a PTP involvement in TE PCD. In addition, CsA inhibited TE formation (data not shown), and also blocked the PCD triggered by BetA, further implying a PT pore involvement in TE differentiation and possibly PCD. CsA is a cyclic undecapeptide with largely unmodified alkyl side chains, which sits in a hydrophobic pocket in $\mathrm{CpD}$ corresponding to the active site. It is generally assumed that $\mathrm{CpD}$ associates with ANT via the active site and that CsA blocks the pore by preventing this association. ${ }^{36}$ Apoptosis suppression by CsA was correlated with the prevention of mitochondrial dysfunction and caspase activation. ${ }^{27}$ In TEs, CsA may conceivably have additional sites of action and it remains possible that CsA blocks a rate-limiting step downstream of cytochrome $c$ release during TE PCD.

Demonstration of a mitochondrial membrane depolarization, a change in morphology occurring prior to death (vacuole collapse), and induction of a PCD by depolariza- 
tion induced by BetA all point to a role for the mitochondria in TE PCD. In addition, the use of CSA to block death without blocking cytochrome $c$ release suggests that cytochrome $c$ relocation is insufficient to trigger death in these cells. Therefore, the question arises as to what other mitochondrial derived signals may be involved? Reactive oxygen species (ROS) were recently reported to mediate mitochondrial-dependent apoptosis induced by transforming growth factor in fetal hepatocytes, ${ }^{37}$ and may also regulate PCD in TEs. Mitochondrial involvement in apoptosis has recently been consolidated by the finding of other major pro-apoptotic factors in the intermembrane space. These comprise a proportion of certain procaspases, including procaspase- $9,{ }^{38}$ and AIF. ${ }^{8,9,39}$ While plants lack prototypical caspases, they have AIF-like proteins although an apoptotic function has not yet been determined.

\section{Protease activity involved in TE formation}

In animal cells, caspases are essential to many types of animal PCD. Prototypical caspases are not found in plants. Recently, Uren et al. ${ }^{28}$ carried out iterative database searches and identified one family of caspase-like proteins in plants they designated as metacaspases. Type I plant metacaspase have prodomains with a proline-rich repeat motif and a zinc finger motif similar to those of plant hypersensitive response protein LSD-1. Stress-induced caspase activity in plants and prevention of PCD associated with plant hypersensitive response by caspase inhibitors have been reported. ${ }^{40}$ zVAD-fmk is a universal caspase inhibitor of nuclear apoptosis, occurring in intact cells irrespective of the cell type. zVAD-fmk caspase inhibitor can block TE formation. While little effect was found on necrotic cell death (data not shown), it remains to be seen whether this caspase inhibitor blocks putative metacaspases activity or another unknown plant protease. Phytepsins (plant pepsin-type enzymes, family A1) are the only aspartic endopeptidases implicated in plant PCD, and pepstatin is the most commonly used inhibitor of phytepsins. Because pepstatin A (20 and $200 \mu \mathrm{M})$ showed little effect on TE formation and cell death (data not shown), there is probably no phytepsin involvement in TE formation or PCD.

We confirm the qualitative results of McCann et al. ${ }^{41}$ that zFA-fmk was effective at reducing TE formation, but our results stand in contrast in other ways. First, we did not observe any consistent nor conspicuous delay in TE formation although autolysis may have been delayed in a minority of cells. Second, all the inhibitors tested including zVAD-fmk which were used by McCann et al. ${ }^{41}$ reduced TE formation. Since TE formation is affected, it is not possible at this time to distinguish between a proteolytic event involved in differentiation from one triggering PCD. Until it becomes possible to uncouple the differentiation from PCD, we can not definitely assign or eliminate a caspase activity in TE PCD. However, the data shown here and elsewhere do not support the animal apoptosome mechanism in TE PCD.

\section{Materials and Methods}

\section{Zinnia seedling growth and cell culture}

Seeds (Zinnia elegans L. Cv. Green Envy; Stokes Seeds, Buffalo, NY, USA) were sterilized and grown in a growth chamber at $25^{\circ} \mathrm{C}$ and $60 \%$ humidity with $16 \mathrm{~h}$ of daylight $\left(110 \mu\right.$ photons $\left.\mathrm{m}^{-2} \mathrm{~s}^{-2}\right)$ per day. Leaves were collected from 30 plants 10-15 days after sowing, surface sterilized with $10 \%$ bleach, $0.01 \%$ Triton for $1 \mathrm{~min}$, transferred to $2.25 \%$ bleach, $0.01 \%$ Triton $\mathrm{X}-100$ for 5 min with occasional gentle agitation, and rinsed with sterile water three times. The leaves were macerated for $30 \mathrm{~s}$ in medium described by Groover and Jones ${ }^{21}$ using a rheostat-controlled blender (model 7011S, Waring). The isolation and culture of zinnia mesophyl cells were performed as described by Groover et al. ${ }^{20,21}$ Death percentage was calculated by adding Evan's Blue (in water) at a rate of $1: 30$ or fluorescein diacetate (in DMSO) at a rate of $1: 100$. Death rate was calculated as the sum of dead cells and TE versus the total number of cells.

\section{Transmission electron microscopy}

Cells were collected at various time points and prepared for transmission electron microscopy (TEM) by fixation in a phosphate buffered solution (Sorensen's phosphate, $\mathrm{pH} 5.8$ ) of $2.5 \%$ glutaraldehyde with $0.15 \%$ sucrose and $2 \%$ mannitol to maintain proper osmoticum for $12-24 \mathrm{~h}$ at $4^{\circ} \mathrm{C}$. Cells were post-fixed in $2 \%$ osmium tetroxide in the same buffer for $1 \mathrm{~h}$. After rinsing, the cells were exposed to $2 \%$ uranyl acetate (aqueous) and subsequently embedded in $2 \%$ agar. Samples cut from the agar blocks were dehydrated in a graded ethanol series, infiltrated with Spurr's resin, embedded, and cured at $70^{\circ} \mathrm{C}$ for $24 \mathrm{~h}$. Ultra-thin sections were cut and stained with uranyl acetate and Reynold's lead citrate prior to observation in a Zeiss EM10 transmission electron microscope.

\section{Detection of mitochondria depolarization}

The fluorescent dye, lipophilic cationic probe 5,5',6,6'-tetrachloro$1,1^{\prime}, 3,3^{\prime}$-tetraethylbenzimidazo-carbocyanine iodide (JC-1) has been used to analyze $\Delta \psi$ in intact, living cells. ${ }^{42}$ Increased membrane polarization results in the formation of $\mathrm{JC}-1$ aggregate that causes shifts in emitted light from 530 (monomeric form) to $590 \mathrm{~nm}$ (emission of aggregate). JC-1 changes color from green to red/orange as the membrane potential increases. ${ }^{43}$ Mitotracker dye JC-1 was purchased from Molecular Probes (Eugene, OR, USA). Zinnia cell cultures (24-h and $72-\mathrm{h}$ ) were incubated with $10 \mu \mathrm{g} / \mathrm{ml} \mathrm{JC}-1$ for $20 \mathrm{~min}$ at room temperature, then cells were washed with fresh medium three times. Cells were imaged using a Zeiss LSM410 confocal laser scanning microscope equipped with an Argon-Krypton laser (Figure 5A-D). Images were collected using an excitation of $488 \mathrm{~nm}$ to observe green fluorescence (emission BP 515-540) and an excitation of $568 \mathrm{~nm}$ to observe red fluorescence (emission BP 575-640). The resulting two images were combined and overlaid with a brightfield image of the cell to demonstrate the degree of secondary cell wall formation in that particular cell. For experiments involving BetA and CsA treated cells, a Zeiss LSM510 confocal laser scanning microscope was used (Figure $5 \mathrm{E}-\mathrm{J})$. Images were collected using an excitation of $488 \mathrm{~nm}$ to observe green fluorescence (Argon Laser, emission filter LP505) and an excitation of $543 \mathrm{~nm}$ to observe red fluorescence (Green HeNe Laser, emission filter LP 560). To determine whether JC-1 will stain inner membranes after death, 51-h-old cells were either rapidly frozen, or treated with $4 \% p$-formaldehyde or $2 \%$ glutaraldehyde and JC-1 was immediately added as described. 


\section{Detection of DNA fragmentation with TUNEL}

Suspension culture cells $(1 \mathrm{ml})$ were fixed with $4 \%$ formadehyde in fresh medium for $30 \mathrm{~min}$ at room temperature. Cells were washed with fresh medium, incubated in $200 \mu$ l permeabilization solution $(0.01 \%$ Triton X-100 and $0.1 \%$ sodium citrate in PBS) for $5 \mathrm{~min}$ in ice, then were washed with PBS. Samples were incubated in reaction mixture (In situ Cell Death Detection Kit, Fluorescein, Boehringer Mannheim) for $1 \mathrm{~h}$ at $37^{\circ} \mathrm{C}$, washed with PBS, then were suspended in $50 \sim 100 \mu \mathrm{l}$ PBS to continue the assay. Cells were evaluated by fluorescence microscopy (excitation $=470 \mathrm{~nm}$; emission $=510 \mathrm{~nm}$ ). Cells undergoing PCD contained fragmented nDNA which fluoresced green and were expressed as the percentage of total cells, including necrotic cells. A negative control with only terminal transferase instead of TUNEL reaction mixture was included in each experiment. Fixed, permeabilized cells were incubated with DNase I to induce DNA strand breaks to serve as a positive control in each experiment.

\section{Preparation of protein samples}

PCD-induced or control cells were gently homogenized in a mortar and pestle in $200 \mu$ l Buffer A as described by Stein and Hansen ${ }^{22}$ with protease inhibitor cocktail (Boehringer Mannheim. GmBH, Germany). The homogenates were spun at $1000 \times g$ for $10 \mathrm{~min}$ to remove cellular debris and nuclei. The supernatant was spun at $10000 \times g$ for $10 \mathrm{~min}$, yielding a pellet fraction enriched in mitochondria. This pellet was resuspended in buffer $A$. The supernatant was spun at $50000 \times g$ for $1 \mathrm{~h}$. The supernatant recovered from this step contained cytosolic protein.

\section{Immunoblot of cytochrome $\boldsymbol{c}$ and quantification}

Protein was separated on $10 \%$ SDS-PAGE and then transferred to nitrocellulose membrane (Molecular Probe). The membrane was blocked in TBS (10 mM Tris- $\mathrm{HCl}, \mathrm{pH} 8.0,150 \mathrm{mM} \mathrm{NaCl}), 0.05 \%(\mathrm{w} / \mathrm{v})$ Tween 20 (TBS-T) $+5 \%(\mathrm{w} / \mathrm{v})$ dried nonfat milk for $1 \mathrm{~h}$. The mouse monoclonal cytochrome $c$ antibody clone ( $7 \mathrm{H} 8.2 \mathrm{Cl} 2$, PharMingen) was added at a concentration of $1 \mu \mathrm{g} / \mathrm{ml}$ and incubated for $3-4 \mathrm{~h}$. After washing twice $(1 \times 15 \mathrm{~min}, 1 \times 5 \mathrm{~min})$ in TBS-T, the membrane was incubated in goat anti-rabbit HRP conjugate IgG (Amersham) at the dilution of $1: 2000$ for $1 \mathrm{~h}$ at room temperature and washed as indicated above. Labeling was detected by chemiluminescence (Pierce, Rockford) according to the manufacter's instruction. The signal was analyzed by scanning densitometry of the exposed film, and then quantified with IMAGEQUANT (Molecular Dynamics). Commercial and published resources of antibodies to cytochrome $c$ do not recognize native plant cytochrome $c$ as needed for immunolocalization in conjunction with electron and fluorescent microscopes.

\section{Proteases inhibitor effect on TUNEL and TE formation}

Caspase inhibitors were purchased from Kamiya Biochemical Co. (Seattle, WA, USA). z-VAD-fmk in DMSO, z-FA-fmk in DMSO and STI (Sigma, St. Louis, MO, USA) in fresh medium were added to 48 -h-old cells at the indicated final concentrations and cultured for an additional 2 days. Cells were collected and TUNEL and TE percentages were assayed. Control cells were incubated with $1 \%$ DMSO or no addition. Cells treated with $1 \%$ trypsin for $6 \mathrm{~h}$ served as positive controls for TUNEL.

\section{Acknowledgements}

We thank Mr. Brian Jones for technical support. This work was supported by a grant from NSF, Developmental Mechanisms.

\section{References}

1. Steller H (1995) Mechanisms and genes of cellular suicide. Science 267: 1445 1449

2. Newmeyer DD, Farshon CM and Reed JC (1994) Cell-free apoptosis in Xenopus egg extracts: inhibition by $\mathrm{Bcl}-2$ and requirement for an organelle fraction enriched in mitochondria. Cell 79: 353-364

3. Nakai M, Takeda A, Cleary ML and Endo T (1993) The bcl-2 protein is inserted into the outer membrane but not into the inner membrane of rat liver mitochondria in vitro. Biochem. Biophys. Res. Commun. 196: 233-239

4. Zamzami N, Morchetli P, Castedo M, Zanin C, Vayssiere JL, Petit PX and Kroemer G (1995) Reduction in mitochondrial potential constitutes an early irreversible step of programmed lymphocyte death in vivo. J. Exp. Med. 181: 1661-1672

5. Shimizu S, Nartia M and Tsujimoto (1999) Bcl-2 family proteins regulate the release of apoptogenic cytochrome $c$ by the mitochondrial channel VDAC. Nature 399: $483-487$

6. Fulda S, Scaffidi C, Susin SA, Krammer PH, Kroemer G, Peter ME and Debatin KM (1998) Activation of mitochondria and release of mitochondrial apoptogenic factors by betulinic acid. J. Biol. Chem. 273: 33942-33948

7. Zou H, Li Y, Liu X and Wang X (1999) An APAF-1: cytochrome $c$ multimeric complex is a functional apoptosome that activates procaspase-9. J. Biol. Chem. 274: $11549-11556$

8. Susin SA, Zamzami N, Castedo M, Hirsch T, Marchetti P, Macho A, Daugas E, Geuskens M and Kroemer G (1996) Bcl-2 inhibits the mitochondrial release of an apoptogenic protease. J. Exp. Med. 184: 1331-1341

9. Joza N, Susin SA, Daugas E, Stanford WL, Cho SK, Li CY, Sasaki T, Elia AJ, Cheng HY, Ravagnan L, Ferri KF, Zamzami N, Wakeham A, Hakem R, Yoshida H, Kong YY, Mak TW, Zuniga-Pflucker JC, Kroemer G and Penninger JM (2001) Essential role of the mitochondrial apoptosis-inducing factor in programmed cell death. Nature 410: 549-554

10. Von Ahsen O, Waterhouse NJ, Kuwana T, Newmeyer DD and Green DR (2000) The 'harmless' release of cytochrome $c$. Cell Death Differ. 7: 1192-1199

11. Jones AM (2000) Does the plant mitochondrion integrate cellular stress and regulate programmed cell death? Trends Plant Sci. 5: 225-230

12. Green DR and Reed JC (1998) Mitochondria and apoptosis. Science 281 $1309-1312$

13. Kroemer G (1997) Mitochondrial implication in apoptosis. Towards an endoymbiotic hypothesis of apoptosis evolution. Cell Death Differ. 4: 443-456

14. Kroemer G, Zamzami N and Susin AS (1997) Mitochondrial control of apoptosis. Immunol. Today 18: 44-51

15. Vander Heiden MG, Chandel NS, Li XX, Schumacker PT, Colombini M and Thompson CB (2000) Outer mitochondrial membrane permeability can regulate coupled respiration and cell survival. Proc. Natl. Acad. Sci. USA 97: 4666-4671

16. Schendel SL, Xie Z, Montal MO, Matsuyama S, Montal M and Reed JC (1997) Channel formation by antiapoptotic protein Bcl-2. Proc. Natl. Acad. Sci. USA 94: 5113-5118

17. Shimizu S, Ide T, Yanagida T and Tsujimoto Y (2000) Electrophysiological study of a novel large pore formed by Bax and the voltage-dependent anion channel that is permeable to cytochrome c. J. Biol. Chem. 275: 12321-12325

18. Jones AM (2001) Programmed cell death in development and defense. Plant Physiol. 125: $94-97$

19. Martin S, Green D and Cotter T (1994) Dicing with death: dissecting the components of the apoptosis machinery. Trends Biochem. Sci. 19: 26-30

20. Groover A, Dewitt N, Heidel A and Jones AM (1997) Programmed cell death of plant tracheary elements differentiating in vitro. Protoplasma 196: 197-211

21. Groover A and Jones AM (1999) Tracheary element differentiation uses a novel mechanism coordinating programmed cell death and secondary cell wall synthesis. Plant Physiol. 119: 375-384

22. Stein JC and Hansen $G$ (1999) Mannose induces an endonuclease responsible for DNA laddering in plant cells. Plant Physiol. 121:1-9 
23. Balk J, Leaver CJ and McCabe PF (1999) Translocation of cytochrome $c$ from the mitochondria to the cytosol occurs during heat-induced programmed cell death in cucumber plants. FEBS Lett. 463: 151-154

24. Sun YL, Zhao Y, Hong X and Zhai ZH (1999) Cytochrome $c$ release and caspase activation during menadione induced apoptosis in plants. FEBS Lett. 462: $317-321$

25. Xu Y and Hanson M (2000) Programmed cell death during pollination-induced petal senescence in petunia. Plant Physiol. 122: 1323-1333

26. Wen YL and Chase CD (1999) Mitochondrial gene expression in developing male gametophytes of male-fertile and S male-sterile maize. Sex Plant Reprod. 11: $323-330$

27. Sugano $\mathrm{N}$, Ito $\mathrm{K}$ and Murai $\mathrm{S}$ (1999) Cyclosporin A inhibits $\mathrm{H}_{2} \mathrm{O}_{2}$-induced apoptosis of human fibroblasts. FEBS Lett. 447: 274-276

28. Uren AG, O'Rourke K, Aravind L, Pisabarro MT, Seshagiri S, Koonin EV and Dixit VM (2000) Identification of Paracaspases and Metacaspases: two ancient families of caspase-like proteins, one of which plays a key role in MALT lymphoma. Mol. Cell. 6: 961-967

29. Deiss LP, Galinka H, Berissi H, Cohen O and Kimchi A (1996) Cathepsin D protease mediates programmed cell death induced by interferon- $\gamma$, Fas/APO-1 and TNF- $\alpha$. EMBO J. 15: $3861-3870$

30. Ye ZH and Varner JE (1996) Induction of cysteine and serine proteases during xylogenesis in Zinnia elegans. Plant Mol. Biol. 30: 1233-1246

31. Minami A and Fukuda H (1995) Transient and specific expression of a cysteine endopeptidase associated with autolysis during differentiation of zinnia mesophyll cells into tracheary elements. Plant Cell. Physiol. 36: 1599-1606

32. Manicini M, Anderson BO, CaldwellE, Sedghinasab M, Paty PB and Hockenbery DM (1997) Mitochondrial proliferation and paradoxical membrane depolarization during terminal differentiation and apoptosis in a human colon carcinoma cell line. J. Cell. Biol. 138: 449-469

33. Martinou I, Desagher S, Eskes R, Antonsson B, Andr E, Fakan Sand Martinou JC (1999) The release of cytochrome cfrom mitochondria during apoptosis of NGFdeprived sympathetic neurons is a reversible event. J. Cell. Biol. 144: 883-889

34. Von Ahsen O, Renken C, Perkins G, Kluck RM, Bossy-Wetzel E and Newmeyer DD (2000) Preservation of mitochondrial structure and function after Bid- or Baxmediated cytochrome c release. J. Cell. Biol. 147: 809-822
35. Pisha E, Chai H, Lee IS, Chagwedera TE, Farnsworth NR, Cordell GA, Beecher CW, Fong HH, Kinghorn AD, Brown DM, Wani MC, Wall ME, Hieken TJ, Das Gupta TK and Pezzuto JM (1995) Discovery of betulinic acid as a selective inhibitor of human melanoma that functions by induction of apoptosis. Nat. Med. 1: $1046-1051$

36. Crompton $M$ (1999) The mitochondrial permeability transition pore and its role in cell death. Biochem. J. 341: 233-249

37. Herrera B, Alvarez AM, Sanchez A, Fernandez M, Roncero C, Benito M and Fabregat I (2001) Reactive oxygen species (ROS) mediates the mitochondrialdependent apoptosis induced by transforming growth factor (beta) in fetal hepatocytes. FASEB J. 15: 741-751

38. Susin SA, Lorenzo HK, Zamzami N, Marzo I, Brenner C, Larochette N, Prevost MC, Alzari PM and Kroemer G (1999) Mitochondrial release of caspase-2 and -9 during the apoptotic process. J. Exp. Med. 189: 381-394

39. Marchetti P, Castedo M, Susin SA, Zamzami N, Hirsch T, Macho A, Haeffner A, Hirsch F, Geuskens M and Kroemer G (1996) Mitochondrial permeability transition is a central coordinating event of apoptosis. J. Exp. Med. 184: 11551160

40. del Pozo O and Lam E (1998) Caspases and programmed cell death in the hypersensitive response of plants to pathogens. Curr. Biol. 8: 1129-1132

41. McCann MC, Stacey NJ and Roberts K (2000) Targeted cell death in xylogenesis. In Programmed Cell Death in Animals and Plants, Bryant JA, Hughes SG and Garland JM, eds (Oxford: Scientific Publishers Ltd) pp. 193-201

42. Cossarizza A, Baccarani Contri M, Kalashnikova G and Franceschi C (1993) A new method for the cytofluorimetric analysis of mitochondrial membrane potential using the J-aggregate forming lipophilic cation $5,5^{\prime}, 6,6^{\prime}$-tetrachloro$1,1^{\prime}, 3,3^{\prime}$-tetraethylbenzimidazolcarbocyanine iodide (JC-1). Biochem. Biophys. Res. Commun. 197: 40-45

43. Salvioli S, Ardizzoni A, Franceschi C and Cossarizza A (1997) JC-1, but not DiOC6(3) or rhodamine 124, is a reliable fluorescent probe to assess $\Delta \psi$ changes in intact cells: implications for studies on mitochondrial functionality during apoptosis. FEBS Lett. 411: 77-82 